\title{
Metallic Axe Causing Cranial Injury: A Case Report.
}

\author{
Dipak Kumar Yadav', Sadaf Saleem Sheikh ${ }^{2}$ \\ ${ }^{1}$ Nobel Medical College and Teaching Hospital, Department of Neurosurgery, Kanchanbari, Biratnagar, \\ Nepal, ${ }^{2}$ Punjab Medical College, Faislabad, Pakistan \\ Correspondence: \\ Dr Dipak Kumar Yadav \\ Department of Neurosurgery, Nobel Medical College and Teaching Hospital \\ Email:dpkdv04@gmail.com
}

\begin{abstract}
Axe as the weapon holds features of both sharp and blunt forces. Physical assault in our community is generally noticed but with such unique weapon is not frequently heard. Homicidal inflicting injury to the head is common in physical assault. Many cases are being admitted with a history of head injury with blunt objects and sharp objects but very few cases are being documented in literature about the use of axe as a weapon. Here is presented a case of 46-year-old right handed male patient form a village in Siraha was brought to the emergency department with the history of physical assault with sharp edged metallic axe.
\end{abstract}

Key words: metallic axe, head injury, cranial injury

$\mathrm{T}$ The axe is a simple machine, which is a type of wedge. Dual inclined surface reduces the effort to chop the wood. Pressure concentration at the blade splits the wood into two parts. The handle of the axe acts as a lever that increases the force at the cutting edge. It has been used for centuries to harvest timber, shape, split and cut wood, and also as a weapon. The axe has many forms and specialized uses but the one that is frequently available in our community has head which is made of metal and handle are generally made of wood. Axe as the weapon holds features of both sharp and blunt forces.

Physical assault in our community is generally noticed but with such unique weapon is not frequently heard.

Homicidal inflicting injury to the head is common in physical assault. Many cases are being admitted with a history of head injury with blunt objects and sharp objects but very few cases are being documented in literature about the use of axe as a weapon.

\section{Case description}

A case of 46-year-old right handed male patient form a village in Siraha was brought to the emergency department of Nobel Medical College and Teaching Hospital by their family members with the history of physical assault with sharp edged metallic axe. Following which there was a

59

Date submitted: 14/ 11/2019

Date accepted: 26/12/2019 history of loss of consciousness and vomiting. Patient was taken to the nearby health care center where immediate first aid was given and referred to a higher center.

Upon receiving at the emergency department the patient was conscious but agitated. His blood pressure(BP) was $140 / 90 \mathrm{~mm}$ of $\mathrm{Hg}$, pulse rate of $136 \mathrm{bpm}$, respiratory rate of $26 \mathrm{bpm}$ and Spo $294 \%$ in room air. His Glasgow coma scale (GCS) on arrival was spontaneous eye opening, confused and obeys commands. His bilateral pupils were $3 \mathrm{~mm}$ and reactive to light. After initial assessment and resuscitation, detailed examination was performed. Cervical tenderness was not present. Cranial nerves examinations were grossly normal. On Motor examination, strength in left biceps, triceps, iliopsoas, gluteal, quadriceps, hamstring and ankle flexor and extensor muscles 4/5. Strength in comparable muscle groups on right was $5 / 5$. Sensory examination shows no obvious clinical findings. Deep tendon reflexes were normal. Examination of the other systems reveals normal findings. There was no obvious sign of cerebrospinal fluid leak. Local examination revealed lacerated and split scalp wound on right temporo-parietal region of approximately measuring $7 \mathrm{~cm} \times 2 \mathrm{~cm} \times 2 \mathrm{~cm}$. There was no active bleeding present. Wound was contaminated.

Wound was thoroughly washed and compressive bandage was applied over the head. There were no

egneuro Volume 02, Issue 01, 2020 DOI: https://doi.org10.3126/egn.v2i1.27471 
other obvious clinical findings in secondary survey. Computed tomography of the head was performed which showed right parietal bone comminuted fracture extending upto left temporal bone with bony defect, right Parietal lobe hemorrhagic contusions with perilesional edema, left subdural hematoma, pneumocephalus, midline shift, and overlying soft tissue swelling.

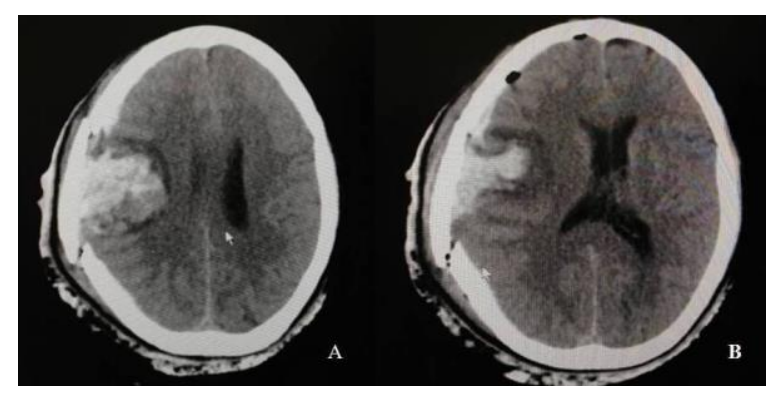

Figure 1(A, B): Right Parietal lobe hemorrhagic contusions with perilesional edema, left subdural hematoma, pneumocephalus midline shift

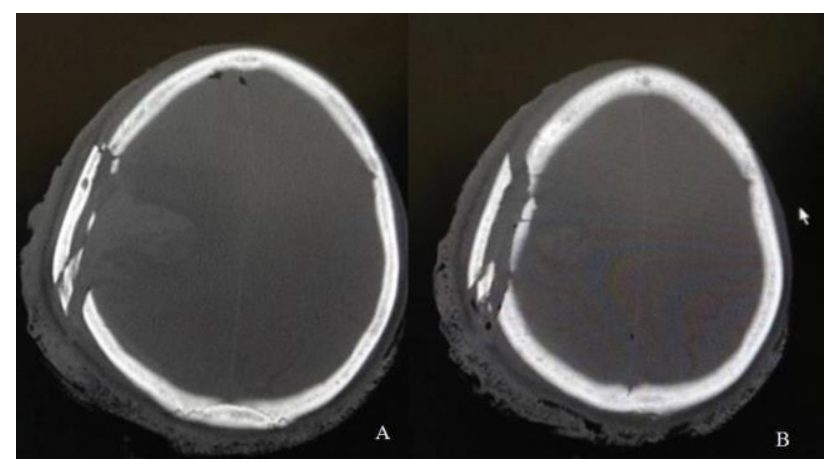

Figure 2(A,B): Right parietal bone comminuted fracture extending upto left temporal bone with bony defect and overlying soft tissue swelling

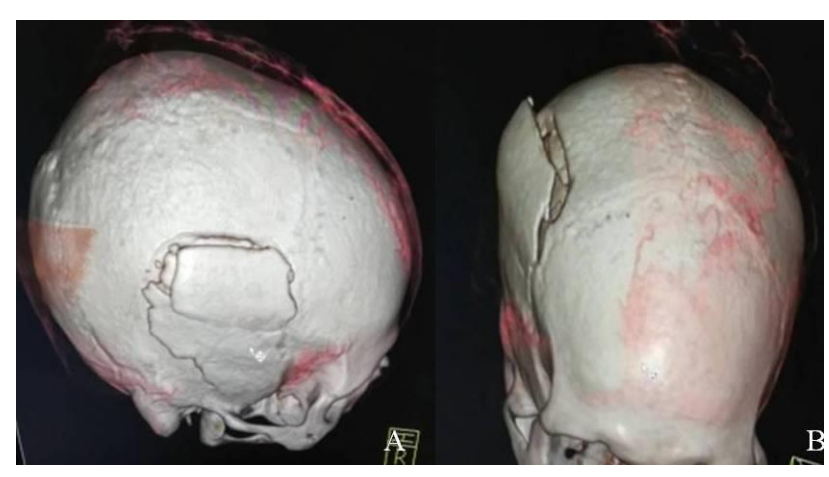

Figure 3(A, B): 3D reconstruction of the CT head.

Preoperatively diagnosed as right parietal bone compound comminuted fracture with underlying parietal lobe contusion with left subdural hematoma with pneumocephalus with midline shift. Patient preoperative hemoglobin was $12.3 \mathrm{gm} / \mathrm{dl}$, total WBC of $16,400 /$ cumm with $85 \%$ neutrophils and other blood parameters were within normal limits. Patient was started on antibiotics and prophylactic antiepileptic.

Patient underwent emergency surgery. Perioperative findings were multiple parietal bony fragments, dural tear, right parietal lobe superficial and deep contusion and multiple active bleeding sites. Bleeding was secured and subdural along with contusion was evacuated. Dural tear was repaired. Large bony fragment was replaced and fixed to nearby bony structures. His immediate postoperative GCS was E1VTM3 and bilateral pupil was $2 \mathrm{~mm}$ reactive. His postoperative hemoglobin was $9.6 \mathrm{mg} / \mathrm{dl}$ and total WBC was $15,500 / \mathrm{cu} \mathrm{mm}$. Antibiotic and antiepileptic were continued postoperatively.

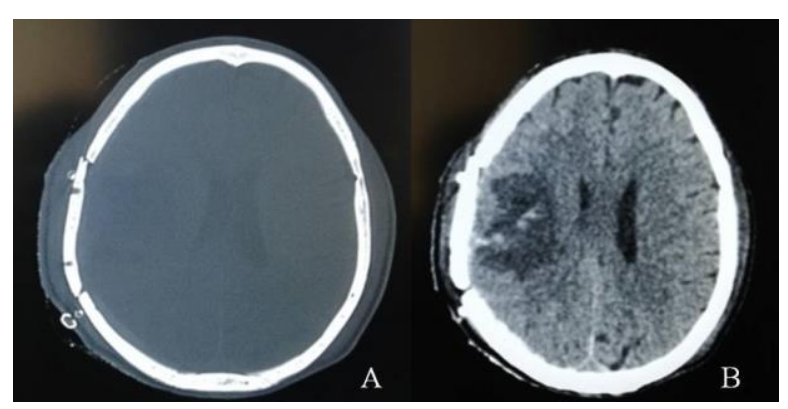

Figure 4(A, B): Postoperative CT- head

Patient was discharged after one week of hospital stay. At the time of discharge, muscle power as per MRC is 5/5 in upper and lower limbs of right side and $4 / 5$ in upper and lower limb of left side.

During the course of hospital stay his vitals were stable and patient never had a seizure. Patient Glasgow Outcome Score was 4 out of 5.

\section{Discussion}

Physical assault is one form of social violence that contributes towards incapacity, psychosocial issues, loss of life, and different health associated morbidities. Violence is outlined as, "the intentional use of physical force or power, vulnerable or actual, against oneself, another person or against a gaggle or community, that 
either ends up in or has high probability of leading to injury, death, psychological hurt, maldevelopment or deprivation. WHO report of 2002 on violence states that social violence results in premature mortality and 90th of this mortality happens in the low and middle income countries. ${ }^{1}$ Nepal follow same patter in term of occurrence of physical assault as seen in different region of the world with local and socio-cultural differences. ${ }^{2}$

In a study by Subba et al., physical assault's median age was 29 years and female had higher age in contrast to male. ${ }^{2}$ Surprisingly, in a study by Basotho, traditional sticks were used in $55 \%$ of cases due to easily availability. ${ }^{3}$ Availability of the axe could be the reason for the choice of weapon in this case.

In a study by Subba, among head injury, contusion (28.7\%) was the commonest type of injury followed by incised wounds $(25.7 \%)$. wooden stick was most commonly used weapon and followed by kicks and punches ${ }^{2}$ Similar finding was observed in study by Hocking where open wounds and contusions was the most common type of injuries. ${ }^{4}$ As seen in different studies, the thorax remains the commonest site of injuries in sharp force ${ }^{5}$ in contrast to the head in blunt force. ${ }^{6}$ Head and neck injury accounted for $78 \%$ for males in the study by Brink et al. ${ }^{7}$

Moreover, because the victims usually fall down or becomes unconscious once receiving blows to head, the aggressor is discouraged to communicate more blows. In distinction to the present, sharp force is sometimes delivered with intention to cause death of the victim and to confirm death, multiple attacks are created sometimes, thereby involving more than one region. ${ }^{8}$ In this case no other injuries were noted. Majority $(80 \%)$ of homicide occur at the residence of the victims. ${ }^{9}$

\section{Conclusion}

Nepal being a developing country physical assault is commonly noticed in community. Mostly locally available weapons or objects are used for such incidence. Physical assault cases with axe are not encountered commonly in hospital. Axe can cause huge injury that could be life threatening as it has property of both blunt as well as sharp weapon and which is easily available in home. Such assaults can only be minimized through public awareness.

\section{Declaration of patient consent}

Consent was taken from patient's son.

\section{Conflicts of interest}

None

\section{References}

1. World report on violence and health. Geneva: World Health Organization;2002.

2. Subba S, Binu V, Menezes RG, Kumar V, Rana MJJof, medicine 1. Physical assault related injuries in Western Nepal-a hospital based retrospective study. 2010; 17(4):203-8.

3. der Stuyft Van PJTJotm, hygiene. Interpersonal violence: patterns in a Basotho community. 1993; 96(2):93-9.

4. Hocking MJJotRSoM. Assaults in south east London. 1989; 82(5):281-4.

5. Rouse DJM, Science, Law t. Patterns of stab wounds: a six year study. 1994; 34(1):67-71.

6. Gudjonsson GH, Petursson HJM, Science, Law t. Some crimonolgical and psychiatric aspects of homicide in Iceland. 1982; 22(2):91-8.

7. Brink O, Vesterby A, Jensen JJI. Pattern of injuries due to interpersonal violence. 1998; 29(9):705-9.

8. Ormstad K, Karlsson T, Enkler L, Law B, Rajs JJJoFS. Patterns in sharp force fatalities-a comprehensive forensic medical study. 1986; 31(2):529-42.

9. Kominato Y, Shimada I, Hata N, Takizawa H, Fujikura TJM, Science, Law t. Homicide patterns in the Toyama prefecture, Japan. 1997; 37(4):316-20. 